\title{
Manejo de antiagregantes y anticoagulantes en procedimientos intervencionistas de dolor crónico
}

\author{
M. Herrero Trujillano, J. Insausti Valdivia, A. Mendiola de la Osa y E.M. Pellejero Collado \\ Unidad del Dolor. Hospital General Universitario de Ciudad Real. SESCAM. Ciudad Real
}

Herrero Trujillano M, Insausti Valdivia J, Mendiola de la Osa A, Pellejero Collado EM. Manejo de antiagregantes $y$ anticoagulantes en procedimientos intervencionistas de dolor crónico. Rev Soc Esp Dolor 2015; 22(3): 126-133.

\begin{abstract}
Interventional pain physicians usually face situations were, the patients that are going to be under an interventional procedure, are undergoing an antiplatelet or anticoagulant therapy. Bleeding complications can be catastrophic when we talk about deep blocks and interventions into the spinal canal. However, the risk of thromboembolic events increases with the improper discontinuation of antiplatelet and anticoagulant drugs in those patients.

Nowadays, there are no algorithms or updated recommendations on the handling of those patients in the chronic pain area. The goal of this article is to offer some recommendations on how to use, in a safety way, those drugs depending on the type of intervention and patient, with the objective of minimizing the risk of bleeding complications without increasing the risk of thromboembolic events. To do this, the latest news on the use of drugs which alters the hemostasis in regional anesthesia and other chronic pain technics had been reviewed, including different guides on the perioperative management (ASRA, ESA, SEDAR, etc.).

There is fair evidence that the risk of thromboembolic phenomenon increases on those patients whom discontinues their antiplatelet therapy, and that this risk is even higher than the risk of epidural hematomas on those patients whom continues with their treatment, even though both risks are significant. There is also good evidence of the incidence of spontaneous
\end{abstract}

Recibido: 01-04-14

Aceptado: 15-06-14 epidural hematomas, associated or not to a traumatic puncture, in patients with or without an antithrombotic therapy.

Those spontaneous epidural hematomas are more likely associated to favorable factors such as: Excessive manipulation, the use of larger gauge needles, the use of catheters, procedures into the cervical spinal canal, elderly patients, and vascular and anatomical abnormalities of the patient.

There is a less conservative tendency about intervals of discontinuation of antiplatelet drugs in high risk patients ( 3 days for Aspirin ${ }^{\circledR}, 5$ days for clopidogrel), while the use of heparin and classic oral anticoagulants practically has not changed.

Recently, new oral anticoagulants, not included in most of these guides, have appeared (dabigatran, rivaroxaban and apixaban) with a higher security profile than Sintrom ${ }^{\circledR}$, usually without the need of heparin bridging therapy after discontinuation of the drug. The inconvenience is that there is limited evidence about its perioperative use. That is why it's suspension is actually based on its strict pharmacokinetic and pharmacodynamics characteristics ( 2 days, being higher if there exists a decline in renal function).

Key words: Antiplatelet. Anticoagulant. Therapy. Chronic pain.

\section{RESUMEN}

Los especialistas en dolor crónico nos enfrentamos habitualmente a situaciones en las que los pacientes que van a ser sometidos a procedimientos intervencionistas están bajo tratamiento antiagregante o anticoagulante. Las complicaciones hemorrágicas pueden ser catastróficas cuando se trata de bloqueos profundos y técnicas dentro del canal espinal. Sin embargo, el riesgo de eventos tromboembólicos aumenta con la suspensión inadecuada de los fármacos antiagregantes y anticoagulantes en estos pacientes. A día de hoy no existen unos algoritmos o recomendaciones actualizadas para el manejo de estos pacientes en el área de dolor crónico. El objetivo de este documento es ofrecer unas recomendaciones de seguridad sobre el manejo de estos fármacos según 
el tipo de intervención y el tipo de paciente, con el objetivo de minimizar el riesgo de complicaciones hemorrágicas sin aumentar el riesgo de eventos tromboembólicos. Para ello, se han revisado las últimas publicaciones sobre el manejo fármacos que alteran la hemostasia en anestesia regional y otras técnicas de dolor crónico, incluyendo diferentes guías de manejo perioperatorio (ASRA, ESA, SEDAR...). Existe buena evidencia en el incremento del riesgo de eventos tromboembólicos en pacientes que suspenden la terapia antiagregante, y que este riesgo es mayor que el de la incidencia de hematomas epidurales en pacientes que continúan con el tratamiento, si bien ambos riesgos son significativos. Así mismo, existe buena evidencia de la incidencia de hematomas epidurales espontáneos asociados o no a punción traumática, en pacientes con o sin terapia antitrombótica. Estos hematomas epidurales espontáneos están asociados a factores favorecedores como la manipulación excesiva, el uso de agujas de mayor calibre, el uso de catéteres, los procedimientos sobre el canal cervical, la edad avanzada, y las anormalidades vasculares y anatómicas del paciente. Hay una tendencia menos conservadora hacia los intervalos de interrupción de los fármacos antiagregantes en pacientes de alto riesgo (Aspirina ${ }^{\circledR} 3$ días, clopidogrel 5 días), si bien el manejo de heparinas y anticoagulantes orales clásicos prácticamente no ha cambiado. Recientemente han surgido nuevos anticoagulantes orales no contemplados en muchas de estas guías (dabigatran, rivaroxaban, apixaban) con mayor perfil de seguridad que el Sintrom ${ }^{\circledR}$, habitualmente sin necesidad de terapia puente con heparinas tras la suspensión del fármaco. El inconveniente es que existe escasa documentación en la literatura sobre su manejo perioperatorio, por lo que su suspensión se basa actualmente en sus características farmacocinéticas y farmacodinámicas estrictas ( 2 días, siendo mayor si existe insuficiencia renal).

Palabras clave: Antiagregantes. Anticoagulantes. Terapia. Dolor crónico.

\section{INTRODUCCIÓN}

Una parte clave en la especialidad de dolor crónico es la utilización de procedimientos invasivos para diagnosticar y tratar patologías dolorosas. Muchos pacientes sometidos a estas técnicas están bajo tratamiento antiagregante o anticoagulante, y aunque el riesgo de sangrado mayor es muy bajo, las consecuencias pueden ser muy graves cuando se trata de bloqueos profundos e intervenciones dentro del canal espinal. Sin embargo, no hay que olvidar que la interrupción inadecuada de los tratamientos antitrombóticos en estos pacientes puede incrementar de forma considerable el riesgo de eventos tromboembólicos, por lo que es necesario que existan guías de consenso actualizadas para el manejo de estos enfermos en procedimientos intervencionistas de dolor.

\section{NECESIDAD DE UNAS GUÍAS ACTUALIZADAS}

La literatura sobre terapia antitrombótica y riesgo de sangrado en intervencionismo de dolor crónico es muy escasa. La mayoría de las recomendaciones desarrolladas hasta ahora no están basadas en una evidencia idónea. Esto es debido a que la mayoría de las publicaciones se fundamentan en estudios observaciones y en series de casos. Además, en nuestro país no existen unas guías actualizadas de consenso específicas para la especialidad de dolor en el manejo de estos fármacos. La última publicación sobre el tema en la Revista de la Sociedad Española de Dolor data de 2001 (1). Existen guías y recomendaciones destacadas sobre el manejo general de antiagregantes y anticoagulantes en el perioperatorio que nos pueden servir de ayuda, tanto en España como en el extranjero, aunque son discordantes en ciertos aspectos (americanas, europeas, nórdicas). Las últimas actualizaciones sobre terapia antitrombótica se publicaron en 2011 en la Revista de la Sociedad Española de Anestesiología y Reanimación (SEDAR), y versaban sobre el manejo perioperatorio de antiagregantes en cirugía electiva (2). Anteriormente, en 2010, se difundieron las últimas actualizaciones de las guías americanas (ASRA) y europeas (ESA) sobre anticoagulación y anestesia regional (3). Desde entonces, se han aprobado nuevos antiagregantes y anticoagulantes orales, no contemplados en muchas de estas guías, y se han publicado revisiones recientes que sugieren una mayor comprensión y acuerdo sobre su manejo en la especialidad de dolor crónico.

\section{TENDENCIA ACTUAL}

Revisando las últimas publicaciones sobre el tema se observa una tendencia menos conservadora hacia los intervalos de interrupción de los fármacos antiagregantes en pacientes de riesgo, si bien el manejo de heparinas y anticoagulantes orales clásicos prácticamente no ha cambiado. Esto es debido a que la literatura clásica ha estado siempre más enfocada al riesgo de hematoma espinal y menos al riesgo de tromboembolismo por parte del paciente. De hecho, Manchikanti y cols. observaron en una reciente encuesta que la mayoría de profesionales del dolor interrumpen la medicación antiagregante aun sabiendo que el mantenimiento de esta es considerada una conducta segura (4). El objetivo de estas revisiones es que los pacientes de riesgo lleguen el día de la intervención con unos niveles de actividad del fármaco lo suficientemente bajos como para no producir un sangrado que comprometa la vida, y lo suficientemente altos como para que al reiniciar el fármaco, este alcance niveles terapéuticos rápidamente. De esta manera se disminuiría el intervalo de tiempo en que el paciente se encuentra sin actividad antitromboembólica eficaz.

Manchikanti y cols. realizaron una revisión bibliográfica extensa sobre el riesgo de sangrado en técnicas intervencionistas de dolor crónico sobre el canal espinal (5). Para ello, revisaron guías, protocolos, estudios observaciones y series de casos publicados en los últimos 46 años. Esta preocupa- 
ción derivaba de estudios donde se demuestra que la interrupción de la Aspirina ${ }^{\circledR}$ (AAS) en la prevención secundaria del síndrome coronario agudo (SCA) multiplica por tres el riesgo de ángor, y que este riesgo es mucho mayor en pacientes portadores de stent (6). La descontinuación prematura del tratamiento con clopidogrel después de un SCA multiplica por 30 el riesgo de aparición de eventos coronarios, que es máximo en los 90 primeros días, respaldando así la teoría de un efecto rebote de la reactividad plaquetaria (7). Tras 18.000 procedimientos neuroaxiales con 12.000 hallazgos que incluían unos 3.000 pacientes, estos investigadores observaron que no existe una prevalencia significativa de efectos adversos entre pacientes que suspenden la terapia antiagregante y los que continúan con ella. Es cierto que el mantenimiento de antiagregantes se puede asociar a mayor sangrado pero sin incrementar la mortalidad, excepto en cirugía intracraneal y dentro del canal espinal.

Los resultados de esta revisión concluyen que existe buena evidencia en el incremento del riesgo de eventos tromboembólicos en pacientes que suspenden la terapia antiagregante, y que este riesgo es mayor que el de la incidencia de hematomas epidurales en pacientes que continúan con el tratamiento, si bien ambos riesgos son significativos. Existe buena evidencia de la incidencia de hematomas epidurales espontáneos asociados o no a punción traumática en pacientes con o sin terapia antitrombótica. Estos hematomas epidurales espontáneos están asociados a factores favorecedores como la manipulación excesiva, el uso de agujas de mayor calibre, el uso de catéteres, los procedimientos sobre el canal cervical, la edad avanzada, y las anormalidades vasculares y anatómicas del paciente (espondilosis, espondilitis anquilosante, estenosis de canal, enfermedad de Paget). Sin embargo, la evidencia es limitada para la discontinuación de fármacos como clopidogrel o prasugrel y la reducción del riesgo de hematomas epidurales.

\section{RECOMENDACIONES SEGÚN EL TIPO DE INTERVENCIÓN}

No todos los procedimientos intervencionistas en dolor crónico son de alto riesgo hemorrágico y requieren de la suspensión de antiagregantes y anticoagulantes (Tabla I). Existe evidencia clara en la literatura de que procedimientos intracraneales y dentro del canal espinal (punciones epidurales interlaminares) se asocian a riesgo de hematomas con aumento de la morbimortalidad. La mayoría de los hematomas espinales producen sintomatología a los pocos días de la intervención, sugiriendo que el sangrado se asocia más frecuentemente a una lesión del plexo venoso epidural más que a un sangrado arterial (8). Al contrario, este riesgo es mínimo en intervenciones fuera del canal espinal, como los bloqueos radiculares extraforaminales o la radiofrecuencia de facetas, por lo que teóricamente no sería necesaria una conducta tan restrictiva. Sin embargo, en la zona cervical existe un riesgo aumentado por la proximidad de la arteria vertebral, cuya punción accidental se asocia a complicaciones neurológicas catastróficas. Proce-

TABLA I. RIESGO DE COMPLICACIONES HEMORRÁGICAS SEGÚN EL TIPO DE INTERVENCIÓN EN PACIENTES ANTIAGREGADOS O ANTICOAGULADOS

Alto riesgo de complicaciones hemorrágicas graves

- Intervenciones a nivel intracraneal (ganglio de Gasser, esfenopalatino, glosofaríngeo)

- Intervenciones a nivel cervical profundo (raíces dorsales, facetas, ganglio estrellado)

- Bloqueos epidurales transforaminales a cualquier nivel

- Bloqueos epidurales interlaminares a cualquier nivel

- Bloqueos caudales

- Implante de catéteres epidurales e intradurales

- Implante de electrodos epidurales

- Bloqueos profundos (plexo celiaco, plexo simpático lumbar, psoas por vía posterior)

Moderado riesgo de complicaciones hemorrágicas graves

- Bloqueo diagnóstico/radiofrecuencia pulsada extraforaminal de raíces lumbares

- Neurolisis de ramos sensitivos de los nervios femoral y obturador

- Bloqueo/radiofrecuencia pulsada de nervios intercostales

Bajo riesgo de complicaciones hemorrágicas graves

- Bloqueo/radiofrecuencia convencional de facetas torácicas y lumbares

- Bloqueo/radiofrecuencia pulsada de nervios periféricos (supraescapular, ilioinguinal, occipi-tal, cluneal...)

- Bloqueo/radiofrecuencia pulsada intraarticular

- Bloqueo miofascial 
dimientos intervencionistas a este nivel deberían realizarse con suspensión previa de fármacos que alteren la hemostasia (radiofrecuencia del ramo medial y de raíces cervicales, bloqueo del ganglio estrellado, etc.).

Los bloqueos profundos en pacientes con terapia antitrombótica se asocian a hematomas retroperitoneales de difícil control. Aunque no existen investigaciones que examinen la frecuencia y severidad de estas complicaciones hemorrágicas, en la literatura hay casos publicados con estas complicaciones, siendo las más frecuentes tras bloqueos del simpático lumbar y del psoas por vía posterior $(9,10)$.

Las intervenciones sobre nervios periféricos y las técnicas miofasciales e intraarticulares no se relacionan con alto riesgo de sangrado, si bien habrá que valorar su mantenimiento cuando existan estructuras adyacentes que puedan aumentar las complicaciones hemorrágicas, como por ejemplo la neurolisis de ramos sensitivos de los nervios obturador y femoral, por su estrecha relación con el paquete vascular femoral, o la radiofrecuencia de nervios intercostales, por el riesgo de posible hemotórax.

\section{CONSIDERACIONES ESPECÍFICAS EN PACIENTES PORTADORES DE STENT}

Los pacientes sometidos recientemente a colocación de stent necesitan de doble antiagregación durante un tiempo determinado, ya que la suspensión inadecuada del tratamiento se asocia a trombosis del stent en más de 1/3 de los casos. Si creemos necesaria dicha interrupción, recomendamos antes consultar con el cardiólogo.

Anteriormente hemos hablado del incremento del riesgo tromboembólico con la interrupción prematura de AAS o clopidogrel, por lo que habrá que seguir ciertas recomendaciones (17):

- No suspender la doble antiagregación antes de que hayan transcurrido 4-6 semanas tras la colocación de un stent convencional, ni antes de 6-12 meses tras la colocación de un stent farmacoactivo (idealmente 12 meses). Si existe alto riesgo de trombosis del stent, la doble antiagregación se mantendrá más de 1 año ininterrumpidamente.

- En el caso de un stent carotideo, conviene no suspender la doble antiagregación antes de que hayan transcurrido al menos 4 meses del postoperatorio; y para portadores de otros stents intracraneales no carotideos, 1 mes.

\section{RECOMENDACIONES EN PROCEDIMIENTOS INTERVENCIONISTAS DE DOLOR CON ALTO RIESGO DE COMPLICACIONES HEMORRÁGICAS}

Horlocker y cols. publicaron una revisión sobre el manejo de antiagregantes, anticoagulantes y anestesia regional en 2011 (11). Posteriormente, Manchikanti y cols. revisaron las últimas actualizaciones sobre el manejo perioperatorio de terapia antiagragante y anticoagulante en distintas guías (ASRA, ESA, belgas y nórdicas) (5). Oprea y Popescu realizaron en 2013 una revisión del manejo perioperatorio de antiagregantes nuevos y clásicos desde un punto de vista farmacocinético y farmacodinámico (12). De todas estas publicaciones se pueden sacar las conclusiones que se exponen a continuación.

\section{Manejo de antiagregantes (Tabla II)}

- En prevención primaria prevalece el riesgo de sangrado sobre el riesgo tromboembólico, por lo que los tratamientos antiagregantes se pueden suspender con un intervalo de tiempo razonable.

- En prevención secundaria, se recomienda mantener al menos un antiagregante hasta el día de la intervención, idealmente AAS $100 \mathrm{mg}$. En caso de intolerancia o alergia a AAS se puede optar por antiinflamatorios no esteroideos (AINE) con propiedades antiagregantes (flurbiprofeno, naproxeno), si bien su efecto es mucho menor. Las heparinas también se han utilizado clásicamente, pero su actividad sobre las plaquetas es escasa por lo que no se recomiendan. Algunos autores hablan de la "falsa sensación de seguridad" con estas terapias puente, ya que se abusa del tiempo de interupción del antiagregante, retrasando así el reinicio del tratamiento. Lo ideal es mantener la terapia puente lo mínimo posible para que el descenso de los niveles de actividad antitromboembólica del fármaco principal no llegue a suponer un aumento significativo del riesgo de tromboembolismo.

- Los AINE y AAS a dosis bajas no incrementan el riesgo de hematoma epidural espinal y no son una contraindicación para técnicas intervencionistas en pacientes de alto riesgo tromboembólico. Burger y cols. demostraron mediante un metaanálisis en diferentes tipos de cirugía no cardiaca, que el riesgo hemorrágico se multiplica por 1,5 si se mantiene el tratamiento con AAS. Sin embargo, este riesgo mayor no se acompaña de complicaciones hemorrágicas graves, con la excepción de la neurocirugía en la que un pequeño sangrado a nivel intracraneal puede tener resultados catastróficos, y tal vez en la cirugía de próstata. Estos autores concluyen que sólo cuando el riesgo hemorrágico supere al riesgo de complicaciones cardiovasculares estaría justificado interrumpir la AAS (13).

- AAS a dosis altas (300 mg) y la combinación de varios fármacos antiagregantes (clopidogrel + AAS) deben ser considerado su mantenimiento basados en el juicio clínico del riesgo individual y beneficios de 
TABLA II. MANEJO DE ANTIAGREGANTES EN TÉCNICAS DE DOLOR CRÓNICO CON ALTO RIESGO DE COMPLICACIONES HEMORRÁGICAS

\begin{tabular}{|c|c|c|c|}
\hline$A A P$ & Suspensión & Reinicio & Terapia puente \\
\hline AINE & No & - & - \\
\hline AAS 100 mg/24 h $\left(\right.$ Adiro $\left.^{\circledR}\right)$ & No & - & - \\
\hline AAS 300 mg/24 h (Adiro $^{\circledR}$, Tromalyt $\left.^{\circledR}\right)$ & 3 días (2-5) & $24 \mathrm{~h}$ & AAS $100 \mathrm{mg}$ \\
\hline Triflusal $300 \mathrm{mg} / 12 \mathrm{~h}$ (Disgren $\left.{ }^{\circledR}\right)$ & 3 días (2-5) & $24 \mathrm{~h}$ & Triflusal $300 \mathrm{mg} / 24 \mathrm{~h}$ si alto riesgo \\
\hline Dipiridamol (Aggrenox ${ }^{\circledR}$ ) 300-400 mg/24 h & $24 \mathrm{~h} \mathrm{si}+\mathrm{AAS}$ & $24 \mathrm{~h}$ & AAS $100 \mathrm{mg}$ \\
\hline Cilostazol 100 mg/12 h $\left(\right.$ Pletal $^{\circledR}{ }^{2}$ Ekistol $\left.^{\circledR}\right)$ & $48 \mathrm{~h}$ & $24 \mathrm{~h}$ & No \\
\hline Clopidogrel 75 mg/24 h (Plavix $^{\circledR}$, Iscover $\left.{ }^{\circledR}\right)$ & 5 días (5-7) & $24 \mathrm{~h}$ & AAS $100 \mathrm{mg}$ \\
\hline Ticlopidina $250 \mathrm{mg} / 12 \mathrm{~h}\left(\right.$ Tiklid $\left.^{\circledR}\right)$ & 5-7 días & $24 \mathrm{~h}$ & AAS $100 \mathrm{mg}$ \\
\hline Ticagrelol $90 \mathrm{mg} / 12 \mathrm{~h}$ (Brilique ${ }^{\circledR}$ ) & 3-5 días & $24 \mathrm{~h}$ & AAS $100 \mathrm{mg}$ \\
\hline Prasugrel 5-10 mg/ 24h (Effient $\left.{ }^{\circledR}\right)$ & 7 días & $24 \mathrm{~h}$ & AAS $100 \mathrm{mg}$ \\
\hline Abcifimab (Reopro $\left.{ }^{\circledR}\right)$ & 24-48 horas & No datos & No \\
\hline Tirofiban $\left(\right.$ Agastrat $\left.^{\circledR}\right)$ & 8 horas & No datos & No \\
\hline Epifibatide (Integrilín ${ }^{\circledR}$ ) & 8 horas & No datos & No \\
\hline
\end{tabular}

*Si alergia o intolerancia a AAS, flurbiprofeno $50 \mathrm{mg} / 12 \mathrm{~h}$ (suspender 12 horas antes de intervención).

la técnica, por lo que en intervenciones de alto riesgo de sangrado es preferible mantener únicamente AAS $100 \mathrm{mg}$ los 2-5 días previos.

- Triflusal es un fármaco similar a la AAS, pero con mayor tolerabilidad gastrointestinal. Se puede suspender 2-5 días antes, y si existe alto riesgo tromboembólico por parte del paciente se puede mantener, pero a dosis mínimas (300 mg/24 h).

- Inhibidores de los receptores de ADP (tienopiridinas): el riesgo de sangrado con tienopiridinas es más frecuente que con la Aspirina ${ }^{\circledR}$. Existe evidencia limitada en la interrupción de estos fármacos y la prevención de hematomas epidurales. Clopidogrel tiene un efecto máximo de inhibición plaquetaria a los 3-7 días de tratamiento, y las guías clásicas como la ASRA recomiendan la interrupción de 7 a 10 días antes. Perakis y cols. evidenciaron en un estudio la recuperación de hasta el $70 \%$ de la función plaquetaria a los 5 días de interrupción del tratamiento (14). Aunque se trata de un estudio pequeño, podría estar justificado ese intervalo de suspensión para pacientes de alto riesgo tromboembólico, sobre todo si se trata de portadores de stent. Ticlopidina tiene un menor perfil de seguridad que el clopidogrel, con una interrupción del tratamiento de unos 7 días.

- Prasugrel y ticagrelol son nuevos inhibidores de receptores ADP con mejor perfil de seguridad para el tratamiento del SCA y la angioplastia. Estudios comparativos con clopidogrel evidencian menor riesgo de eventos isquémicos debido a un rápido inicio de acción y una mayor inhibición plaquetaria (15). Actualmente se indican por delante del clopidogrel en el tratamiento del SCA, según las guías europeas de cardiología (nivel de evidencia IA). Los intervalos de interrupción previos a la punción neuroaxial en pacientes de riesgo son de 7 días para prasugrel y de 3 a 5 días para ticagrelol.

- Inhibidores de la fosfodiesterasa como dipiridamol y cilostazol no parecen incrementar el riesgo de hematoma epidural y no son una contraindicación para estas técnicas. No obstante, dipiridamol se suele asociar a AAS ya que su efecto antiagregante en monoterapia ocurre a dosis que provocan hipotensión por vasodilatación, por lo que en terapia combinada habría que suspenderlo 24 horas antes. Con respecto al cilostazol, la evidencia en la literatura sobre su mantenimiento es escasa, y debido a que su indicación se reduce principalmente a pacientes con enfermedad vascular periférica no susceptible de cirugía, parece razonable su interrupción 48 horas antes, como se describe en las guías de la SEDAR.

- Inhibidores de la glicoproteína IIb/IIIa (abciximab, tirofiban, eptifibatide) se utilizan en perfusión continua intravenosa durante la fase aguda del SCA, por lo que es muy raro su manejo en el área del dolor crónico. Actualmente su uso se ha reducido significativamente, aunque recientemente se están publicando series de casos que abogan por utilizar eptifitabide y tirofiban como terapia puente en pacientes de alto riego, no contemplados en nuestras guías pero sí por la Sociedad 
de Cirugía Torácica (16) y en las guías de Australia y Nueva Zelanda. Estos fármacos se asocian a trombocitopenia, por lo que habría que pedir un recuento plaquetario antes de realizar una intervención.

- El reinicio de la terapias antiagregantes tras procedimientos de alto riesgo de compilaciones hemorrágicas puede realizarse entre las 6 y 24 horas siguientes, idealmente 24 horas, sobre todo si ha habido dificultades de la técnica (varios intentos de punción neuroaxial, aspirado de sangre por catéter, etc.).

\section{Manejo de anticoagulantes (Tabla III)}

- Las heparinas continúan con los mismos intervalos de interrupción y reinicio del tratamiento para procedimientos sobre el canal espinal y bloqueos profundos.

- La heparina no fraccionada (HNF) necesita de una interrupción de 4 horas y una actividad de TTPa ratio segura $(<1,5)$. Si el tratamiento es prolongado (más de 5 días), conviene realizar un recuento de plaquetas ya que en un $25 \%$ de los casos se asocia a trombocitopenia. El reinicio del tratamiento se realizará cuando haya transcurrido 1 hora de la técnica, o 6 horas en caso de punción hemática.

- Las heparinas de bajo peso molecular (HBPM) requieren de un mayor intervalo de interrupción debi- do a una vida media de eliminación más prolongada. Cuando se utilizan a dosis profilácticas, 12 horas son suficientes para la realización de la técnica, con un reinicio a las 6 horas del postoperatorio (o 24 horas si punción hemática). Cuando se utilizan a dosis terapéuticas, se necesitan 24 horas de interrupción previa al procedimiento y otras 24 horas para el reinicio.

- Fondaparinux es un inhibidor del factor X similar a las HBPM. El riesgo de hematoma espinal asociado no está del todo claro. El intervalo de interrupción del fármaco es de 36 horas y el reinicio se realiza a las 6 horas de la punción neuroaxial, o bien a las 12 horas de retirada de catéter epidural. En pacientes con insuficiencia renal estos intervalos son más prolongados.

- Los antagonistas de la vitamina K son considerados una contraindicación absoluta para la realización de técnicas neuroaxiales hasta que el INR se encuentre en valores normales $(<1,5)$. El Sintrom ${ }^{\circledR}$ se debe suspender de 3 a 5 días antes, con un reinicio 24 horas después de la técnica si no ha habido complicaciones.

\section{NUEVOS ANTICOAGULANTES ORALES Y ANESTESIA NEUROAXIAL}

En los tres últimos años han surgido nuevos anticoagulantes orales (NACO) -dabigatran, rivaroxaban, apixa-

TABLA III. MANEJO DE ANTICOAGULANTES EN TÉCNICAS DE DOLOR CRÓNICO CON ALTO RIESGO DE COMPLICACIONES HEMORRÁGICAS

\begin{tabular}{|c|c|c|c|}
\hline$A C O$ & Suspensión & Reinicio & Terapia puente \\
\hline HNF & $\begin{array}{c}\text { 4h } \\
\text { TTPa Ratio }<1,5\end{array}$ & $\begin{array}{c}1 \mathrm{~h} \\
\text { (6 h px hemática) }\end{array}$ & No \\
\hline HBPM/24 h (dosis profiláctica) & $12 \mathrm{~h}$ & $\begin{array}{c}6 \mathrm{~h} \\
\text { (24 h px hemática) }\end{array}$ & No \\
\hline HBPM/12 h (dosis terapéutica) & $24 \mathrm{~h}$ & $24 \mathrm{~h}$ & No \\
\hline Fondaparinux (Arixtra ${ }^{\circledR}$ ) & $36 \mathrm{~h}$ & $\begin{array}{c}6 \mathrm{~h} \\
12 \mathrm{~h} \text { si retirada catéter }\end{array}$ & No \\
\hline Acenocumarol (Sintrom ${ }^{\circledR}$ ) & $\begin{array}{l}\text { 3-5 días } \\
\text { INR }<1,5\end{array}$ & $24 \mathrm{~h}$ & HBPM \\
\hline Dabigatran 200-300 mg/ 24 h (Pradaxa $\left.{ }^{\circledR}\right)$ & $\begin{array}{c}48 \mathrm{~h} \\
(4 \text { DIAS si CCr < 50) } \\
\text { TP si dudas }\end{array}$ & $24 \mathrm{~h}$ & $\begin{array}{c}\text { No } \\
\text { HBPM si alto riesgo }\end{array}$ \\
\hline Rivaroxaban $10-20 \mathrm{mg} / 24 \mathrm{~h}\left(\right.$ Xarelto $\left.^{\circledR}\right)$ & $\begin{array}{c}48 \mathrm{~h} \\
\text { (3 días si CCr <30) } \\
\text { TP si dudas }\end{array}$ & $24 \mathrm{~h}$ & $\begin{array}{c}\text { No } \\
\text { HBPM si alto riesgo }\end{array}$ \\
\hline Apixaban 2,5-5 mg/ 12 h (Eliquis $\left.{ }^{\circledR}\right)$ & $\begin{array}{c}48 \mathrm{~h} \\
(\mathrm{CCr}<30 \text { no estudios })\end{array}$ & $24 \mathrm{~h}$ & $\begin{array}{c}\text { No } \\
\text { HBPM si alto riesgo }\end{array}$ \\
\hline Sulodexida $12 \mathrm{mg} / 8 \mathrm{~h}$ (Aterina ${ }^{\circledR}$ ) & $24 \mathrm{~h}-48 \mathrm{~h}$ & $24 \mathrm{~h}$ & No \\
\hline
\end{tabular}


ban- con el objetivo de contrarrestar las limitaciones del Sintrom ${ }^{\circledR}$. Tienen la ventaja de que su dosificación es fácil, no necesitan de controles de laboratorio frecuentes, y se asocian a menos interacciones y a menor riesgo de sangrado gastrointestinal e intracraneal según los últimos estudios que los comparan con warfarina en el tratamiento de pacientes con fibrilación auricular. Sus inconvenientes son la falta de reversores estudiados a día de hoy, los poco específicos controles de laboratorio y la muy escasa literatura respecto al manejo perioperatorio.

Dabigatran es un inhibidor directo de la trombina, mientras que rivaroxaban y apixaban tienen actividad anti factor Xa, similar a las HBPM. Alcanzan su efecto máximo en pocas horas y su eliminación es renal. Los controles de laboratorio son poco específicos, aunque un tiempo de protrombina (TP) normal excluiría niveles significativos de estos fármacos.

Su utilización ha sido aprobada para la profilaxis del postoperatorio de prótesis total de rodilla y cadera, la profilaxis tromboembólica de fibrilación auricular no valvular en pacientes con factores de riesgo (hipertensión arterial, diabetes mellitus, edad avanzada, disfunción ventricular, antecedentes de eventos tromboembólicos), y para el tratamiento del tromboembolismo. Las recomendaciones de su uso preferente son la intolerancia o alergia a antagonistas de la vitamina $\mathrm{k}$, el antecedente de ictus hemorrágico, el alto riesgo de hemorragia intracraneal, el mal control del INR pese al cumplimiento terapéutico, y las complicaciones tromboembólicas a pesar de un INR en rango.

Para reducir el riesgo de sangrado y de eventos tromboembólicos en pacientes con NACO, se recomienda posponer los procedimientos neuroaxiales si:

- Un evento tromboembólico ha ocurrido en los 3 meses previos (IAM, ictus, TVP, etc.)

- Un evento hemorrágico mayor ha ocurrido en los 3 meses previos (descenso de 2 puntos de hemoglobina, transfusión de 2 o más concentrados de hematíes, sangrado dentro de un órgano).

- La paciente está embarazada o han transcurrido menos de 6 meses de postparto.

Benzon y cols. realizaron en 2013 una revisión sobre los NACO y la anestesia regional (18). Debido a que no existen publicaciones que avalen el manejo perioperatorio de estos fármacos, el tiempo de interrupción y su reinicio están basados en sus características farmacocinéticas. Dabigatran necesita de mínimo 2 días de interrupción del fármaco para la realización de una anestesia neuroaxial o de un bloqueo profundo (4 días si $\mathrm{CCr}<50 \mathrm{ml} / \mathrm{min}$ ). Rivaroxaban requiere de 2 días de interrupción ( 3 días si $\mathrm{CCr}<30 \mathrm{ml} / \mathrm{min}$ ). El reinicio del tratamiento puede realizarse a las 24 horas de la técnica, si no ha habido complicaciones. No es necesario realizar una terapia puente con HBPM como ocurre con el Sintrom ${ }^{\circledR}$, salvo en casos de alto riesgo.

\section{OTROS ANTITROMBÓTICOS}

Recientemente están surgiendo nuevos fármacos con actividad antitrombótica, indicados en el tratamiento de pacientes con enfermedad vascular periférica. Sulodexida es un hipolipemiante con actividad anti-factor Xa. En comparación con heparina, tiene una vida media más prologada, menor capacidad de alterar la hemostasia normal y menor riesgo de sangrado (19). Sin embargo, no existen a día de hoy estudios sobre su manejo perioperatorio, por lo que recomendamos una interrupción previa de 24-48 horas.

\section{CONCLUSIONES}

En el manejo del tratamiento antiagregante y anticoagulante en dolor crónico es imprescindible una evaluación individualizada de cada paciente y de cada procedimiento intervencionista. Los algoritmos de actuación suponen una ayuda en la toma de decisiones, pero siempre deberá prevalecer la decisión tomada por el equipo médico responsable del paciente, una vez valorado el riesgo/ beneficio en cada caso. Se deberá siempre informar adecuadamente al paciente (y/o sus familiares) acerca de la decisión tomada y el riesgo asumido, dejando constancia por escrito en la historia clínica del paciente. Los fármacos antiagregantes se suspenden en prevención primaria, ya que prevalece el riesgo de sangrado. En prevención secundaria, conviene al menos mantener un antiagregante (idealmente AAS) con el objetivo de disminuir el riesgo de eventos tromboembólicos. Los intervalos de suspensión de los demás fármacos antiagregantes se ajustarán de acuerdo con el incremento del riesgo tromboembólico de cada paciente. En pacientes portadores de stent se intentará respetar el mantenimiento de la doble antiagregación para evitar la trombosis del stent. Los anticoagulantes orales son una contraindicación absoluta para técnicas intervencionistas dentro del canal espinal. Son necesarios nuevos estudios que evalúen el riesgo de sangrado para los nuevos anticoagulantes orales. Así mismo, es recomendable la existencia de algoritmos y guías de consenso específicas para el manejo de la terapia antiagregante y anticoagulante en el área del dolor crónico.

\footnotetext{
CORRESPONDENCIA:

Manuel Herrero Trujillano

Unidad del Dolor

Hospital General Universitario de Ciudad Real

C/ Obispo Rafael Torija, s/n

13005 Ciudad Real

e-mail: manuelherrerotrujillano@gmail.com
} 


\section{BIBLIOGRAFÍA}

1. Llau JV, de Andrés J, Gomar C, Gómez-Luque A, Hidalgo F, Sahagún J, Torres LM. Fármacos que alteran la hemostasia y técnicas regionales anestésicas y analgésicas: recomendaciones de seguridad (foro de consenso). Rev Soc Esp Dolor $2001 ; 8: 337-48$

2. Sierra P, Gomez-Luque A, Castillo J, Llau JV. Guía de práctica clínica sobre el manejo perioperatorio de antiagregantes plaquetarios en cirugía no cardiaca. Rev Esp Anestesiol Reanim 2011;58:1-16.

3. Horlocker TT, Wedel DJ, Rowlingson JC, Enneking FK, Kopp SL, Benzon HT, et al. Regional Anesthesia in the Patient Receiving Antithrombotic or Thrombolytic Therapy: American Society of Regional Anesthesia and Pain Medicine EvidenceBased Guidelines. Reg Anesth Pain Medicine 2010;35:64-101.

4. Manchikanti L, Benyamin RM, Swicegood JR, Falco FJE, Datta S, Pampati V, et al. Assessment of practice patterns of perioperative management of antiplatelet and anticoagulant therapy in interventional pain management. Pain Physician 2012;15:E955- E968.

5. Manchikanti L, Falco F, Benyamin RM, Caraway DL, Kaye $\mathrm{AD}$, Standiford Helm II, et al. Assessment of bleeding risk of interventional techniques: A best evidence synthesis of practice patterns and perioperative management of anticoagulant and antithrombotic therapy. Pain Physician 2013;16:261-318.

6. Biondi-Zoccai GG, Lotrionte M, Agostoni P, Abbate A, Fusaro M, Burzotta F, et al. A systematic review and metaanalysis on the hazards of discontinuing or not adhering to aspirin among 50,279 patients at risk for coronary artery disease. Eur Heart J 2006;27:2667-74.

7. Ho PM, Peterson ED, Wang L, Magid DJ, Fihn SD, Larsen GC, et al. Incidence of death and acute myocardial infarction associated with stopping clopidogrel after acute coronary syndrome. JAMA 2008;299:532-539. Erratum in: JAMA 2008;299:2390.

8. Moen V, Dahlgren N, Irestedt L. Severe neurological complications after central neuraxial blockades in Sweden 19901999. Anesthesiology 2004;101:950-9.
9. Klein SM, D'Ercole F, Greengrass RA, Warner DS. Enoxaparin associated with psoas hematoma and lumbar plexopathy after lumbar plexus block. Anesthesiology 1997; 87:1576-9.

10. Maier C, Gleim M, Weiss T, Stachetzki U, Nicolas V, Zenz M. Severe bleeding following lumbar sympathetic blockade in two patients under medication with irreversible platelet aggregation inhibitors. Anesthesiology 2002; 97:740-3.

11. Horlocker TT. Regional anesthesia in the patient receiving antithrombotic and antiplatelet therapy. $\mathrm{Br} \mathrm{J}$ Anesth 2011;107:96-106.

12. Oprea AD, Popescu WM. Perioperative management of antiplatelet therapy. Br J Anesth 2013;111:3-17.

13. Burger W, Chemnitius JM, Kneissl GD, Rücker G. Lowdose aspirin for secondary cardiovascular prevention - cardiovascular risks after its perioperative withdrawal versus bleeding risks with its continuation - review and meta-analysis. J Intern Med 2005;257:399-414.

14. Perakis PG, Yunus T, Long G, Cheema ZF, Hammond R, Shanley CJ. Platelet function after cessation of chronic clopidogrel therapy. Am Surg 2012;78:51-6.

15. Wallentin L, Becker RC, Budaj A, Cannon CP, Emanuelsson $\mathrm{H}$, Held C, et al. Ticagrelor versus clopidogrel in patients with acute coronary syndromes. N Engl J Med 2009; 361:1045-57.

16. Ferraris VA, Saha SP, Oestreich JH,et al. 2012 Update to the Society of Thoracic Surgeons guideline on use of antiplatelet drugs in patients having cardiac and noncardiac operations. Ann Thorac Surg 2012;94:1761-81.

17. Chou S, Eshaghian S, Lamer A, Tran H, Dohad S, Kaul $\mathrm{S}$. Bridging therapy in the perioperative management of patients with drug-eluting stents. S Rev Cardiovasc Med 2009;10:209-18.

18. Benzon HT, Avram MJ, Green D, Bonow RO. New oral antcoagulants and regional anesthesia. Br J Anesth 2013; 111:96-113.

19. Andreozzi GM. Sulodexide in the treatment of chronic venous disease. Am J Cardiovac Drugs 2012;12:73-81. 